\title{
VIRTUAL FITTING OF PERSONALISED KNITWEAR BASED ON 3D ANTHROPOMETRY
}

\author{
Anton H. JELLEMA, Maartje van der BIE, Wenjun ZHOU and Toon HUYSMANS \\ Delft University of Technology, Faculty IDE, 2628 CE, The Netherlands
}

\begin{abstract}
With personalised knitwear many of the drawbacks of mass-produced garments can be tackled, e.g. bad fit, overstock, lack of individualisation. A major challenge with personalised knitwear, however, is that the customer can not physically try the garment before purchase. Virtual fitting is an emerging area that has the potential to replace physical fitting. This paper addresses the development of two different procedures for the virtual fitting of a sweater. For online shopping a 3D avatar was used based on Do It Yourself measurements of the body (with the help of statistical based prediction of body measurements). For the in-shop tool a 3D avatar was made based on body measurements taken from the customer by a skilled person or an advisor. To support both tools, a concept design of the interface was made based on Clo3D (design software for fashion) and tested. In both cases the digital garment on the avatar was evaluated by the test customer in terms of fit, the look and feel of the garment and was later (after the 'ordered' garment was knitted) compared with the customer wearing the real knitwear, to evaluate the consumer satisfaction with the final result as well as the user experience about the procedure.
\end{abstract}

Keywords: Online shopping, customised knitwear, Do it Yourself body measurements, virtual visualisation of knitwear, fit mapping.

\section{INTRODUCTION}

The Faculty of Industrial Design Engineering of the Delft University of Technology offers a bachelor's degree education programme and three master programmes. Our students are lectured in ergonomics and learn to design and conduct research in ergonomics. In this paper we describe the development of methods to realize virtual fitting of personalised knitwear based on 3D anthropometrics by two master graduation students in our field lab Ultra Personalised Products and Services (UPPS) [1].

Fashion is still dominated by mass production leading to a need for stock and sales predictions for every brand, model and garment size. Although the knowledge of human size distribution and corresponding patterns has increased, there is still a chance of miscalculation. Pattern makers must deal with measurements, ease and posture at the same time. A prototype of a garment is manufactured and evaluated with test persons [2]. An anthropometric fit test of the garment can be very valuable at this stage [3]. 3D scanning is used in the fashion industry to measure 3D human shapes and sizes, to represent 3D human shape variations in fashion design software with avatars, to do size recommendation and to efficiently produce and distribute fashion. Posture is currently one of the main issues when using 3D human models in fashion design, because posture is difficult to implement due to time-consuming modelling tools [4]. Besides, we see growing numbers of online garments sales, whereby the use of 3D human models also comes into play.

Digital fitting technology based on a digital human model is a good solution for online shopping. It is used to improve shopping value and satisfaction because customers can see how the selected garment looks like and fits. The use of 3D human models for online shopping is already far better than looking at a picture of a model to evaluate if a certain garment is suitable for the customer [5].

The digital model can be based on a 3D scan of the customer (digital clone) or on a parametric human body which represents the user or customer as close as possible (digital twin). It is based on a 3D anthropometric library whereby a human model can be selected and modified by entering certain parameters like height, weight and demographic factors like country, nationality and age [6]. This is more accurate than selecting garment based on size [5]. There are size inconsistencies across brands, leading to uncertainty and to the customer buying the wrong size, especially when shopping online, which results in high rates of returned products.

Companies like triMirror and Clo3D create easy to use applications with avatars based on huge 3D 
databases collected during large-scale surveys involving body scanners. These applications are used for virtual fitting tools for mobile applications and in-store systems (virtual mirror). The problem with the avatars is that they remain the same posture regardless of measurements and that a digital clone, based on a simple body scan, is unreliable and lacks precision (e.g. 3D scan extracted from photos made with a smartphone application) [4]. It is still unclear how this should be used in different situations (online and in shop) and what the benefit of the tools is regarding satisfaction while buying (experience) and after receiving the garment (the fit).

This paper is the next step to advance the procedure of virtual fitting by explaining the development of two different procedures of virtual fitting of a sweater with a digital twin. For online buying of a sweater the avatar was based on Do It Yourself (DIY) measurements of the body [7]. For the in-shop tool (also used to buy a sweater) a 3D digital twin was made based on body measurements taken from the customer by a skilled person or an advisor [8]. For both tools a concept design of the interface based on Clo3D, is made and tested. In both cases the digital garment on the avatar was evaluated by the test customer in terms of virtual fit, the look and feel of the garment and was later (after the 'ordered' garment was knitted) compared with the customer wearing the real knitwear, to evaluate the consumer satisfaction with the final result as well as the user experience about the procedure.

Knitwear is more friendly for the environment compared to the traditional production of clothing, also because knitwear can be unravelled, and the yarn reused. The knitting machine technology is a driver for companies to offer personalised knitwear through online platforms or via physical shops. Confidence in the virtual fitting procedure and satisfaction with the result is very important because personalised knitwear is more expensive than mass-produced garments.

\section{CONSTRUCTING AND EVALUATING A DIGITAL TWIN BASED ON DO IT YOURSELF BODY MEASUREMENTS}

To find out what could be learnt from actual solutions, 3 state of the art online solutions were evaluated with 5 test participants (students) to figure out what can be learnt from the existing websites [7]. The evaluated websites are Zozo (sweater), Fit finder (sweater) and Bivolino (shirt). The most important findings were that the participants wanted to know the garment size created from their own body measurements (height, weight, age for the sweater). It was difficult to answer the question about preferred fit (slim/regular/loose fit) since that is too abstract. Finally, when the system asks limited size information from the participants, it raised doubts and uncertainty, although the size prediction algorithm may reduce the customers effort.

To knit a sweater, the measurement of some of the body dimensions is needed besides the customer's gender and age. But some of these dimensions are difficult to measure oneself: biacromial breadth (shoulder width), arm length, waist back (distance from neck to waist) and scye depth. Therefore, it has been calculated whether the dimensions that are difficult to measure could be predicted, based on the easy to measure DIY dimensions. We used data from CAESAR [9] for these calculations (Age, Weight, Stature, Chest Girth, and Waist Circumference). The prediction is realised through a linear regression model with varying number of input variables. The optimal number of 5 input variables showed more accurate results than if retrieved by DIY measurements (for Waist Back, the optimal number of predictors is 2, i.e. weight and stature). See Figure 1, with on the left a screenshot from the test application, the instructions how to do the measurement of chest girth (chest circumference at scye) and the fields to fill in the results. On the right the virtual sweater on the avatar (virtual twin), with the options to adjust the preferred fit of the sweater. 

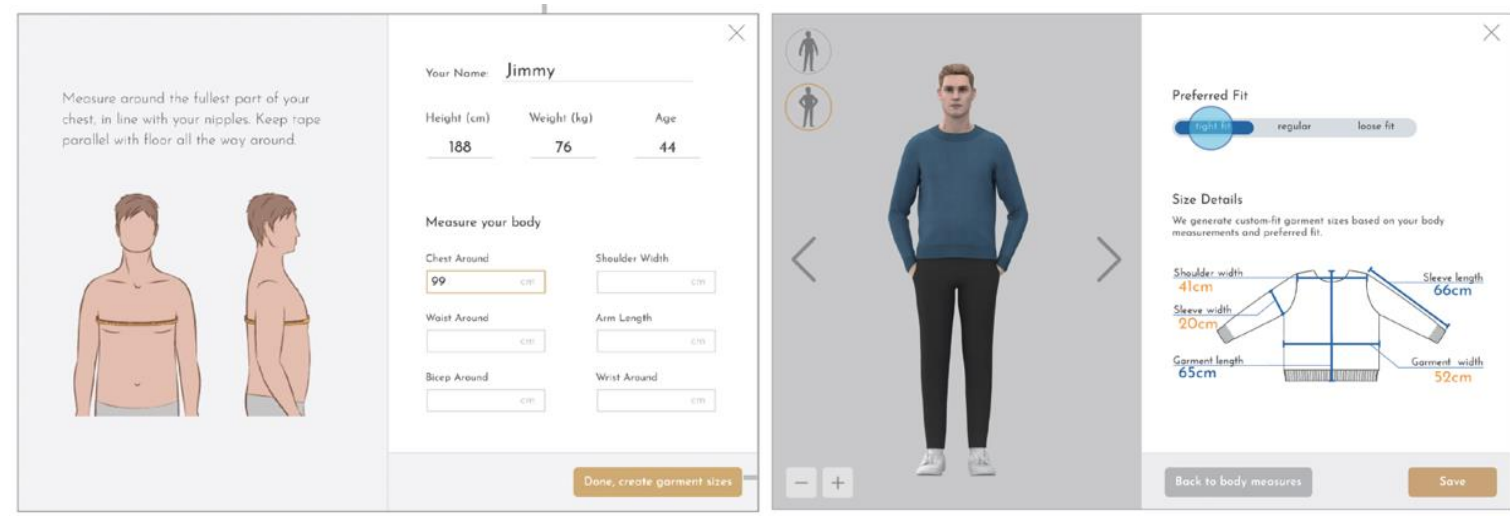

Figure 1. Two screenshots from the test application for online shopping. On the left the DIY measurements can be filled in, on the right the virtual fit of the sweater with options for preferred fit

The test interface, with the personalised avatar from Clo3d, is evaluated by 13 participants. Not all the participants liked the avatar, but 10 people said it boosted their confidence in evaluating the fit of their sweater online. People made mistakes with the DIY measurements and felt insecure about the calculated size of the sweater. That is because they did not know about the prediction calculation and they thought the measurements they had entered, could not be sufficient for a good result. The most important limitation was related to a lack of tactile information from the virtual garment, which was a standard fabric simulation in Clo3D. Besides this evaluation, for two of the participants the sweater was knitted and handed out three weeks later to find out if the 'buyers' were satisfied with the outcome, the real sweater, see Figure 2.
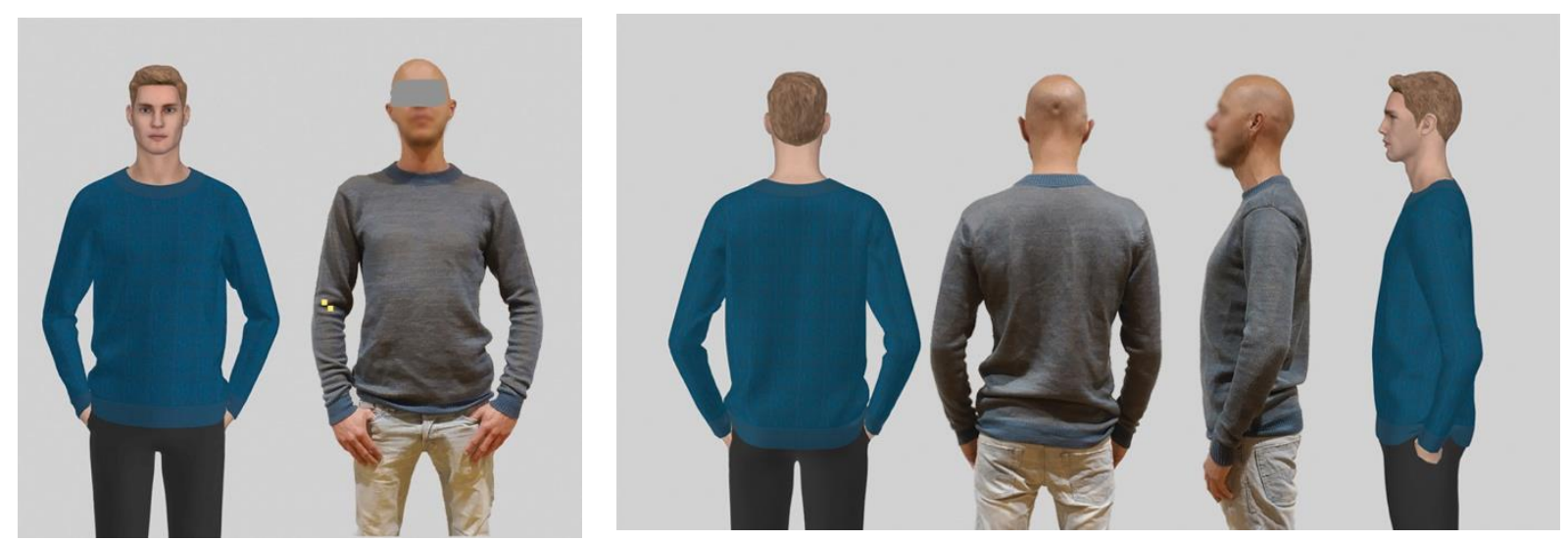

Figure 2. Comparison between virtual fitting and physical try on. The avatar has a thicker neck, more sloped shoulders and looks thinner from the side. The real garment has more folds, a tighter neck and closes more around the waste than the virtual garment

\section{CONSTRUCTING AND EVALUATING A DIGITAL TWIN FOR IN-SHOP USE}

The other approach [8] was to design an application for in-shop use, to visualise a personalised knitwear (sweater). The most important difference with the online application [7] is the fact that the interface is operated by staff, and the staff also takes the body measurements. The avatar is created with the help of Clo3d, with the following parameters: height, weight, chest circumference, waist, hip circumference, arm length and leg length. The accuracy of the Clo3d avatar was compared with a 3D scan from the CAESAR database (digital clone) [6], which showed the necessity to use a maximum number of parameters to create the avatar, see Figure 3. An artist impression of the in-shop virtual mirror is shown in Figure 4. 


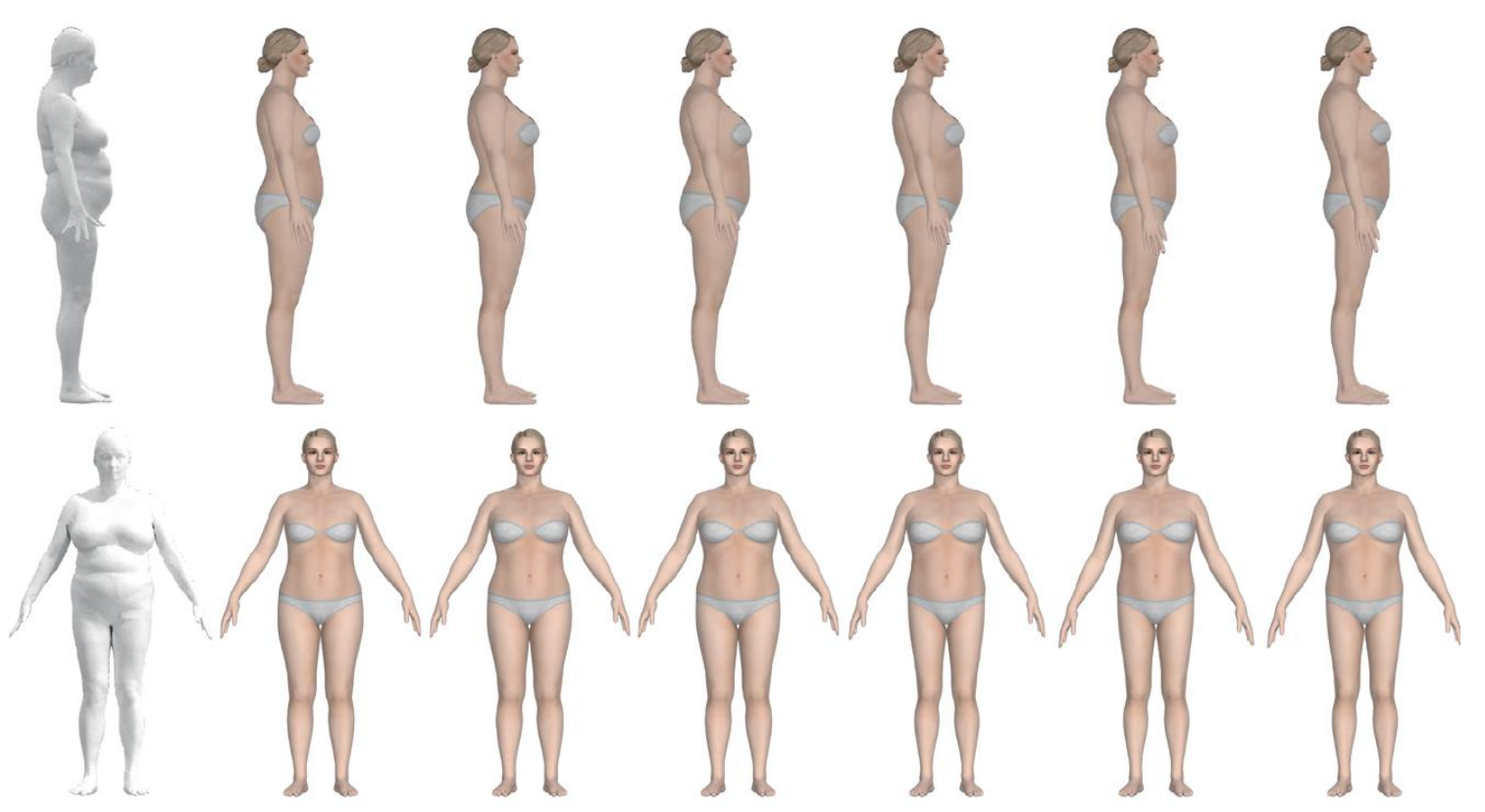

Figure 3. A CAESAR 3D scan compared with the Clo3D avatar, from left to right the avatar is improved (weight + height) + chest circumference + waist circumference + hip circumference + arm length + leg length
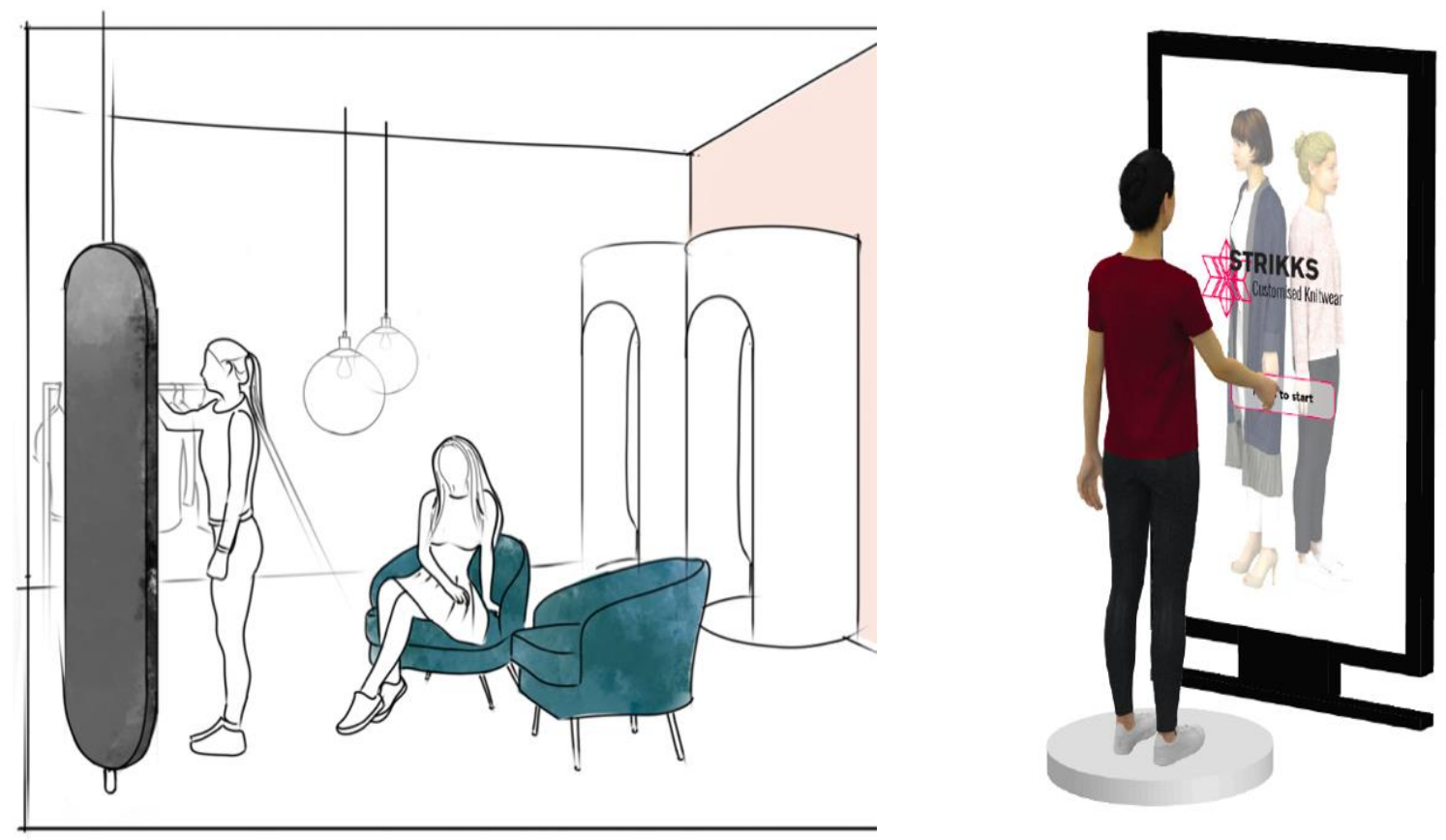

Figure 4. In shop use of the virtual mirror, designed for STRIKKS [8]

The visualisation of a sweater on an avatar created with the Clo3D software was done with 6 female participants, selected from a student population based on the criterium that they had an issue with finding the right clothes in daily life. Subsequently, the sweater was knitted and 4 weeks later it was handed over to the participants for an evaluation.

The posture of the avatar is not always realistic, like it can be seen on the avatar of the participant with the skirt, which leads to an unnatural look, see Figure 5. The neckline looks wider and the sleeves are too long, but this is due to miscalculations in the pattern of the knit wear (participant with skirt). The yellow sweater has also longer sleeved than the virtual sweater, and the drape is slightly different. The overall look is satisfactory. 
The participants said the avatar did not look like themselves (skin colour, face, hair) and the visualisation of the knitwear was unsatisfactory. The software needs to be improved to better simulate how the knitwear drapes around the surface of the body. Further, the participants had doubts about the selection of the sweater (colour, pattern, yarn) and they needed assistance from an advisor [8].
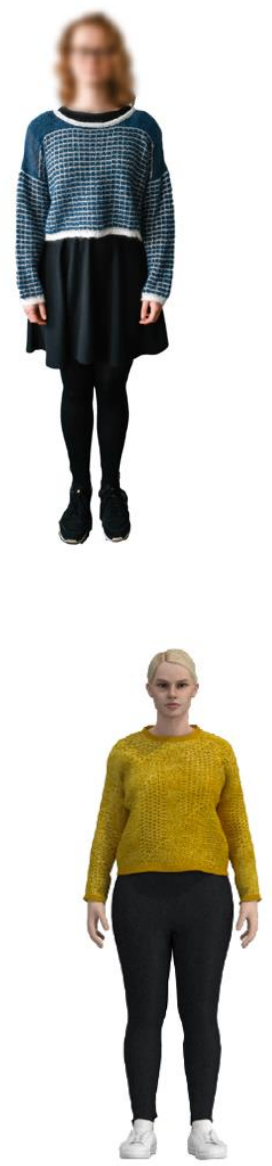
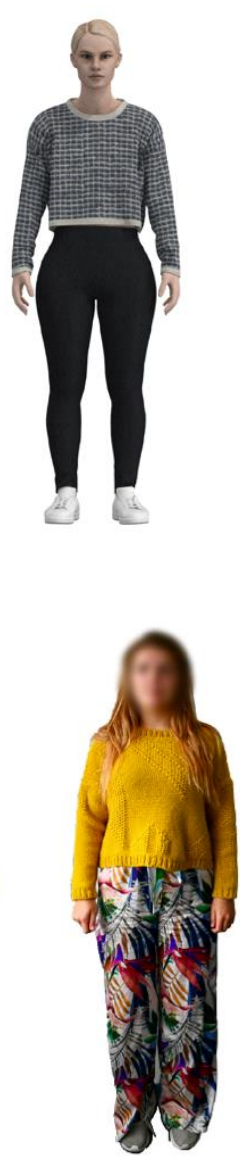
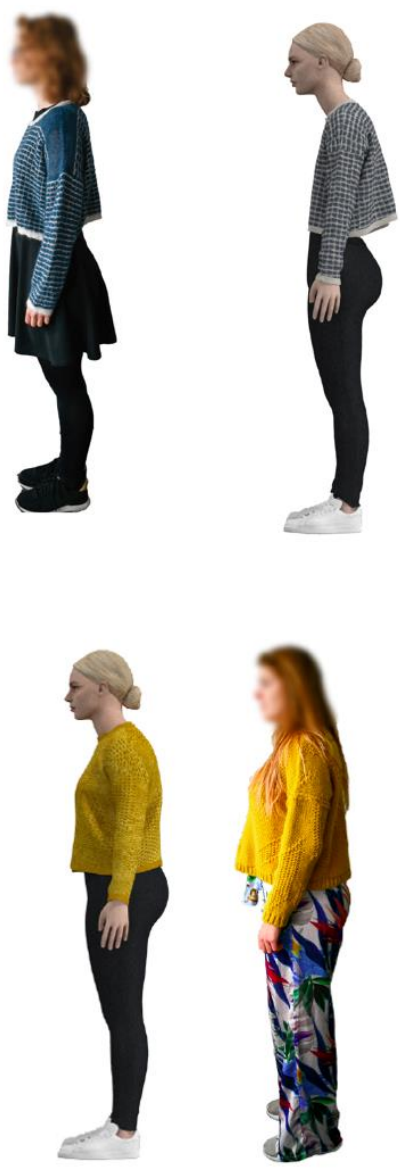

Figure 5. Two participants with the virtual fitted sweater compared with the real sweater

\section{CONCLUSIONS}

The use of avatars for the virtual fitting of clothes is still in the development phase, and the avatars can be improved with regard to body texture, posture, movements and how the materials of the virtual garment can be draped with a natural look around the digital human model (this paper) including how a virtual garment behaves during motion and compression [5]. Concerning online shopping, it has been confirmed that people need to be much more guided and explained throughout the process of body measurements including explanations about sizes prediction based on the given dimensions. Otherwise customers may become unsure and hesitate to purchase. Finally, it becomes clear that the use of avatars is promising for the future, it has advantages for online sales and for the sale of personalised garments in a shop compared to the use of sizing systems.

\section{ACKNOWLEDGMENT}

Both projects [7] and [8] could be realised thanks to a grant from ClickNL [10]. These projects were an opportunity for all parties to widen the scope of industrial design education into the field of fashion. Including the chance to perform statistical calculations to predict human sizes and shapes and to discover how craftmanship of knitting and fashion design can be implemented into the creation of an interface for the sales of personalised knitwear.

\section{REFERENCES}

[1] Delft University of Technology, Faculty IDE. Fieldlab UPPS. Available: http://www.upps.nl/en/ [Accessed on 2020, 20 May]. 
[2] Domingo, J., Simó, A., Ibáñez, M. V., Dura, E., Ayala, G., \& Alemany, S. Towards a mean body for apparel design. Image and Vision Computing, 2016, 52, 88-96. https://doi.org/10.1016/j.imavis.2016.04.016

[3] Bradtmiller, B. Predicting Product Accommodation: The Role of the Anthropometric Fit Test. Procedia Manufacturing, 6th International Conference on Applied Human Factors and Ergonomics (AHFE) Volume 3, 2015, 4464-4471 https://doi.org/10.1016/j.promfg.2015.07.458

[4] Durá-Gil, J.V., Kozomara, Z., Ballester, A., Solves-Camallonga, C., Pierola-Orcero, A. Clothing. In DHM and Posturography (2019) pp 599-611 Scataglini, S. and Paul, G. (Eds.), London, Cambridge, United Kingdom, San Diego, United States: Elsevier.

[5] Kim, H. S., Choi, H. E., Park, C. K., \& Nam, Y. J. Standardization of the size and shape of virtual human body for apparel products. Fashion and Textiles, 2019, 6(1). https://doi.org/10.1186/s40691-019-0187-z

[6] Huysmans, T., Goto, L., Molenbroek, J., \& Goossens, R. DINED Mannequin. Tijdschrift voor Human Factors, 2020, 45(1).

[7] Zhou, W. Virtual fitting of personalized knitwear (MSc graduation project), 2019, Delft University of Technology, The Netherlands.

[8] Bie, van der, M. Designing a user experience to sell personalized knitwear in-store (MSC graduation project), 2019, Delft University of Technology, The Netherlands.

[9] Robinette K. M., Blackwell S., Daanen H., Boehmer M. and S. Fleming, Civilian American and European Surface Anthropometry Resource (CAESAR), 2002, Final Report. Volume 1. Summary, sytronics INC Dayton OH.

[10] ClickNL for creative professionals and enterprising researchers, ClickNL. Available https://www.clicknl.nl/en/ [Accessed on 2020, 5 June]. 